EDUCATION

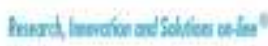

PSYCHOLOGY

$1+0+1$
Electronic Journal of Research

in Educational Psychology

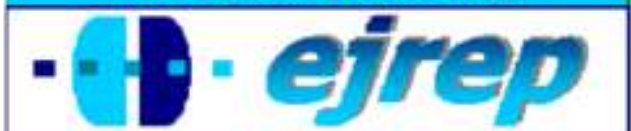

\title{
Relaciones entre aprendizajes escolares y extra-escolares en la investigación educativa. Fundamentos, tendencias y perspectivas.
}

\author{
Cimolai, Silvina ${ }^{1}$; Lucas, Julia Paola ${ }^{2}$ y \\ Pérez, Andrea Verónica ${ }^{3}$
}

${ }^{1}$ Universidad Pedagógica de la Provincia de Buenos Aires (UNIPE) e investigadora Universidad Nacional de Quilmes (UNQ) en el Programa

"Escuela, diferencia e inclusión".

${ }^{2}$ Docente e investigadora en UNIPE e investigadora en UNQ en el Programa "Escuela, diferencia e inclusión".

${ }^{3}$ Docente e investigadora en UNIPE y UNQ en el Programa "Escuela, diferencia e inclusión". Becaria CONICET.

Integrantes de equipo de investigación dirigido por Prof. Ricardo Baquero.

\section{Argentina}

Correspondencia: Silvina Cimolai. CEI-UNQ. Roque Sáenz Peña 352. Bernal. CP: 1876. Pcia. de Buenos Aires. Argentina. Tel 54-11-4365-7100 int 4207. E-mail: silcimolai@gmail.com

(C) Education \& Psychology I+D+i and Editorial EOS (Spain) 


\section{Resumen}

El presente trabajo indaga las aportaciones de investigaciones educativas y psicoeducativas, provenientes de diferentes países -en particular Argentina- que han abordado el problema de las relaciones entre los aprendizajes escolares y los extra-escolares, tomando como base empírica más de cien publicaciones. En primer lugar, analiza las investigaciones de acuerdo a cómo son definidos los problemas de investigación y los tipos de diseños que se priorizan; seguidamente reflexiona sobre los tipos de fundamentación utilizados para justificar la relevancia de los estudios e identifica y realiza un análisis crítico de los objetos culturales que se priorizan en este tipo de estudios; finalmente pone en diálogo los estudios analizados, profundizando la mirada en las continuidades y discontinuidades indagadas en cada caso. Frente a la tendencia observada en estas investigaciones, donde los diversos aprendizajes y contextos son caracterizados en términos de ruptura, o donde las posiciones subjetivas del alumnado son leídas en términos de actividad/pasividad, este trabajo advierte la necesidad de observar con mayor grado de detalle los matices que pudieran presentarse en las relaciones entre los diversos contextos, y concluye que existe un fuerte dinamismo entre los aprendizajes que allí se generan. Asimismo, se analizan las implicancias de que las indagaciones suelen estar definidas a partir de los conocimientos, formas de aprender y contenidos que son tradicionalmente escolares, desestimando la indagación de otros contenidos o aspectos de los aprendizajes que podrían ser relevantes. Otro aspecto sobre el que se reflexiona es la relación directa que hay entre la definición de estos problemas de investigación y el foco en grupos poblacionales provenientes de sectores desfavorecidos o minorías, mostrando el carácter político implícito en el supuesto generalizado de que este tipo de indagaciones podría proveer de conocimientos y estrategias para contribuir a la disminución del fracaso escolar en dichos sectores.

Palabras Clave: Aprendizajes escolares, Aprendizajes extra-escolares, Aprendizaje situado, Investigación educativa.

Recibido: 29/07/10 Aceptación Inicial: 02/09/10 Aceptación Definitiva: 03/03/11 


\title{
Relationships between scholastic and extra-scholastic learning in educational research. Foundations, trends and prospects.
}

\begin{abstract}
This paper analyzes the contributions of educational and psycho-educational research on the debate about the relationships between knowledge and learning practices in school contexts and in out-of-school contexts. It takes into account more than one hundred publications from different countries, focusing on the Argentinean contributions. Firstly, a classification of types of research problems and research designs prioritised by the researchers is presented. Then, arguments used to support this type of studies are identified and critically analysed, as well as types of cultural objects studied in most of the research in the topic. Finally, the continuities and discontinuities highlighted by the studies among learning experiences and knowledge in school and out-of school contexts are presented. It is observed that in most of the research on the topic, there is an emphasis on highlighting differences and discontinuities between school learning and out-of-school learning, and in the subjective positions of people involved in each of the experiences. On the contrary, this paper advices to take into account the nuances and dynamisms of the comparisons not only between learning in school and in everyday life, but also within each of the learning contexts. Another aspect reflected in the paper is the profound relation between the research topics prioritized by the research on the topic and the focus on disadvantaged or minority groups, showing the assumption that taking into account different learning contexts could help improving school achievement of disadvantaged groups.
\end{abstract}

Keywords: School learning, out-of-school learning, situated learning, educational research.

Received: 07/29/10 Initial Acceptance: 09/02/10 Definitive Acceptance: 03/03/11 


\section{Introducción}

El trabajo que aquí se expone forma parte de un estudio dedicado a indagar las contribuciones provenientes de la investigación educativa internacional y local (Argentina) en torno de las continuidades y discontinuidades existentes entre los aprendizajes escolares y los aprendizajes extra-escolares. Se han considerado las producciones que, con un interés educativo, han puesto en relación o en discusión los conocimientos y formas de aprender característicos de ámbitos escolares con aquellos que los niños y niñas desarrollan en otros contextos. La finalidad del trabajo es la de contribuir al desarrollo de una perspectiva conceptual que atienda a las relaciones, tensiones y especificidades de los aprendizajes en diferentes contextos, poniendo el foco en las diferentes categorías teóricas propuestas, en sus objetivos y lineamientos metodológicos, como también en la forma de construir las caracterizaciones de cada aprendizaje, y en las fundamentaciones generalmente utilizadas para justificar la relevancia de este tipo de estudios ${ }^{1}$.

Las investigaciones y producciones analizadas se inscriben en un debate complejo y con larga tradición en el ámbito educativo, acerca de la conveniencia o inconveniencia de incluir en el contexto escolar conocimientos y formas de aprender que los niños y niñas desarrollan en su vida cotidiana, o en otros términos, acerca de la especificidad del aprendizaje escolar con respecto a otros tipos de aprendizajes. El mismo se observa alternadamente tanto en la investigación educativa, en los medios de prensa, como en las mismas reflexiones de actores que trabajan cotidianamente en las escuelas, y en la definición de ciertos lineamientos de políticas educativas. Las discusiones no son sólo acerca de los conocimientos que deberían articularse, o de los modos de lograr esa articulación, sino también acerca de los sentidos y la legitimidad misma de este interés.

Las posiciones quizás más cautelosas con respecto a la legitimidad de la discusión parten de análisis normativos acerca de la función de la escolarización en la sociedad. En este nivel del debate, se sostiene la necesidad de respetar la especificidad que debiera tener una propuesta escolar, como un espacio de formación que brinde a los niños la posibilidad de conocer contenidos y formas de aprender diferentes a las que tienen acceso en su vida cotidia-

\footnotetext{
1 El estudio mencionado es parte de los proyectos de investigación que las autoras integran en la Universidad Pedagógica de la Provincia de Buenos Aires (UNIPE) y en la Universidad Nacional de Quilmes (UNQ) bajo la dirección de Ricardo Baquero, Argentina.
} 
$n^{2}$. En esta misma línea se ubicaría la pregunta acerca de hasta qué punto los intereses de los niños deberían o no definir el curriculum escolar (mencionado por ejemplo en Isbell, 2006). Adicionalmente, para algunos académicos, la validez de promover este tipo de abordajes se enfrentaría además con un dilema ético basado en las relaciones entre las esferas públicas y privadas de las vidas de los niños, y en las tensiones posibles cuando se buscan articular ambas (Hughes, 2006a). En cambio, para autores como Zipin (2009), el dilema ético residiría en las decisiones acerca de lo que posee "valor de aprendizaje" en el contexto escolar.

Cuando se acepta la legitimidad de la posible articulación, el debate se configura entonces en términos de qué es lo que se puede o debe incluir en la escuela, cuál es el status de eso a incluir, y con qué fin. Con respecto a la delimitación de aquello a articular, una primera discusión parece ser acerca de incluir contenidos o modos alternativos de aprender. En ambos casos, el dilema es ¿cuáles serían aquellos conocimientos o estrategias de aprendizaje de la vida extra-escolar que resultan de utilidad o validez en el aprendizaje escolar? En este caso, resulta ilustrativo el debate sostenido por un grupo de docentes en una investigación en Australia (Zipin, 2009), que intentando recuperar los conocimientos de la vida cotidiana de sus alumnos se encontraron con relatos de violencia, crimen, abuso de alcohol y drogas que los llevó a decidir privilegiar la construcción de lo escolar como una zona de seguridad donde los niños y niñas tuvieran la oportunidad de vivenciar otras realidades y preocupaciones. En América Latina, y con otro impacto, se pueden citar las investigaciones de Paradise (1990, 1991, 1994) con alumnos de origen mazahua, quien mostró experiencias escolares diferentes a las tradicionales en tanto las interacciones en las aulas parecían seguir patrones de aprendizaje 'nativos', observables en contextos extra-escolares.

En cuanto a los sentidos de la inclusión de lo extra-escolar en la vida escolar, se observa que generalmente las discusiones alternan entre una inclusión de corte más utilitario para potenciar un mejor rendimiento en un curriculum que no se modifica sustantivamente (Thomson y Hall, 2008); y aquellas que buscarían una inclusión en pos de una modificación radical al curriculum escolar. En la perspectiva más utilitaria, se ubicarían las propuestas que promueven la utilización de situaciones o aprendizajes de la vida cotidiana para potenciar el

\footnotetext{
${ }^{2}$ Por ejemplo, el filósofo Peter Sloterdijk ha cuestionado la propuesta de Ministro de Cultura de Alemania de introducir la música pop en la enseñanza escolar, argumentando que la educación es en sí misma una actividad conservadora y que tiene la responsabilidad de delimitar y transmitir aquellos bienes culturales que la sociedad valora y que desea preservar para las nuevas generaciones (Levy, 2003).
} 
aprendizaje de contenidos escolares ya definidos y que no se modifican (Huddleston, 2003). En la posición que promueve una modificación curricular radical, se pueden mencionar las propuestas que sostienen la inclusión de la cultura popular en un curriculum que es definido como reproductor exclusivo de una cultura de elite. En este sentido, la inclusión de intereses de la vida extra-escolar de los niños y de sus familias de todos los sectores sería una forma de democratizar el curriculum y la escuela, y de promover la preservación y valoración de formas culturales minoritarias (Thomson, 2002).

\section{Metodología}

Se trata de un diseño de investigación donde la fuente primaria de información ha sido el conocimiento académico producido hasta el momento con respecto al tema. En el diseño, se consideraron algunos aportes de las estrategias sistemáticas de síntesis de las investigaciones (Sistematic Research Synthesis), propuestas especialmente en la literatura sajona para organizar y retroalimentar el conjunto de producciones académicas realizadas sobre un problema en particular (véase por ejemplo, Popay, 2006; Gough y Elbourne, 2002). Se siguieron los pasos generales sugeridos para este tipo de procedimientos, a saber: formulación de las preguntas de investigación, identificación y selección de los estudios relevantes, síntesis de los hallazgos y producción de un nuevo conocimiento a partir de ellos (Popay, 2006; Gough, 2004).

Debido tanto al tipo de conocimiento que se buscaba producir (debate conceptual) como al tipo de diseño predominantemente encontrado en las investigaciones sobre nuestro tema de estudio (diseño cualitativo), decidimos focalizarnos en los aportes específicos de la subcorriente sajona llamada meta-etnografía, en tanto la misma se ha mostrado como una estrategia útil de síntesis de conceptualizaciones y hallazgos de investigaciones de corte cualitativo (Britten et al., 2002; Campbell et al., 2006, Popay, 2006). En la investigación educativa, la meta-etnografía fue descripta por primera vez por Noblit y Hare (1988) y estaba principalmente interesada en proveer métodos alternativos de síntesis a la tradicional forma estadística y agregada de análisis. En la meta-etnografía, las interpretaciones y construcciones analíticas de las investigaciones son tratadas como datos, y el método general está basado en el concepto de traducción de metáforas, que implica trasladar y traducir interpretaciones de diferentes estudios unos entre otros, facilitando al investigador la comprensión y transferencia de ideas, conceptos y metáforas a lo largo de diferentes estudios sobre un mismo tema. Según esta perspectiva, la traducción de metáforas entre estudios es la que permite la comparación con- 
ceptual entre las producciones de los mismos, y la producción de conocimientos nuevos basados en esa articulación. Sin embargo, se reconoce la importancia de respetar la situacionalidad de cada estudio, y la particular construcción conceptual que cada uno ha producido. Es importante resaltar que para esta perspectiva, el análisis no se basa en agregar datos de diferentes investigaciones y volverlos representativos de poblaciones más amplias, sino que su aporte reside en identificar instancias empíricas y construcciones conceptuales que al ser puestas en diálogo permitan identificar nudos de problemas que enriquezcan la producción de conocimientos respecto del tema. Como criterios de selección se establecieron:

a) abordajes principalmente empíricos que hubieran atendido a las relaciones entre aprendizajes escolares y extra-escolares, o bien, a la indagación de las formas de aprender en la vida cotidiana con el objeto de informar a las prácticas escolares.

b) producciones provenientes de perspectivas psico o socio-lingüísticas, la psicología cognitiva y la teoría psicogenética, la psicología cultural y producciones antropológicas.

c) por el lugar de inserción de la investigación, se consideró relevar en detalle la producción Argentina, extendiéndose a las investigaciones regionales e internacionales de relevancia que hubieran delineado discusiones conceptuales y empíricas sobre el tema.

En una primera instancia se identificaron más de cien posibles publicaciones a través de una estrategia de búsqueda múltiple y escalonada en la que se priorizó la producción realizada en las últimas dos décadas. En una segunda instancia se seleccionaron las investigaciones más relevantes y se amplió la búsqueda y fichaje a otras investigaciones dentro de cada comunidad académica identificada y a otras más antiguas que aparecían recurrentemente mencionadas en las discusiones. Con esta ampliación buscábamos incluir aquellas investigaciones que son consideradas "clásicas" en la producción sobre el tema.

Finalmente, fueron consideradas 73 publicaciones, de las cuales poco más de la mitad (53\%) correspondían a investigaciones y producciones llevadas a cabo en USA, Europa y Australia; $28 \%$ en Argentina y el resto (19\%) en América Latina. En cuanto al período de tiempo, la mayoría (82\%) fue publicada en las últimas dos décadas (1990-2010). 


\section{Desarrollo}

Tipos de investigaciones

Las investigaciones analizadas fueron organizadas atendiendo a los focos priorizados en el recorte del problema y las áreas analíticas priorizadas en cada investigación. De este modo se identificaron cuatro grandes grupos de problemas (presentados a continuación), teniendo en cuenta que -como se verá- varias de las producciones pueden compartir aspectos de diferentes áreas.

a. Diferencias en los usos del lenguaje (intercambios discursivos, formas de participación e interacción)

En un primer grupo se encuentran las producciones que analizan comparativamente las diferencias en contextos escolares con respecto a otros contextos cotidianos en lo que respecta a los usos del lenguaje, los intercambios discursivos, y las formas de participar y de interaccionar. Un grupo tradicional de indagaciones en este sentido son aquellas que, desde perspectivas socio o psico-lingüísticas, exploraron los usos diferenciales de lenguas o dialectos en contextos cotidianos y escolares (con referentes clásicos como Bernstein, 1971; Labov, 1969; Cazden, 1991; Wells, 1986). Un argumento corriente ha sido que la correspondencia entre el lenguaje standard de la escuela con respecto al de los contextos familiares o comunitarios de los niños, sería uno de los aspectos que signan su éxito escolar (e.g. Thomson, 2002). Por ejemplo, Heath (1983) estableció una correspondencia entre las actividades de comprensión de historias desarrolladas por madres de clase media con sus hijos y aquellas desarrolladas por las profesoras de la escuela.

La idea de que los niños que integran comunidades minoritarias mantienen, respecto del formato escolar, diferentes formas de interacción, como así también diferentes expresiones lingüísticas y cognitivas, suele identificarse con la expresión de 'discontinuidad cultural', tema abordado inicialmente por autores como Philips (1972; 1982) y Heath (1983), quienes han dado cuenta de distintas formas de organización narrativa y de participación en espacios escolares y fuera de ellos. Por ejemplo, a partir del trabajo realizado en una reserva indígena, Philips identificó una particular estructura de participación en las aulas, mientras que advirtió la ausencia de estas estructuras en los contextos familiares y comunitarios de los alumnos, lo 
que a su entender daba lugar a malentendidos entre éstos y los docentes, que eran tradicionalmente interpretados por los referentes de la escuela en términos de dificultad o resistencia por parte de los estudiantes.

En Argentina, poniendo el foco en los intercambios lingüísticos y en los patrones de interacción, Borzone y Rosemberg (2000) han analizado las interacciones verbales y los marcos de conocimientos que niños y niñas pertenecientes a la comunidad kolla ponen de manifiesto tras su participación en contextos familiares o locales y en los contextos escolares, con el objeto de explorar las causas probables de las dificultades en la escuela y elaborar una propuesta de alfabetización intercultural y bidialectal. En la misma línea, Gandulfo (2007) se ha ocupado de analizar los usos y significaciones del guaraní acorrentinado por parte de los pobladores de San José, en la Provincia de Corrientes, donde el ámbito privilegiado de trabajo etnográfico ha sido la escuela. Asimismo, en una escuela situada en la periferia de la capital tucumana, Prado, Canigia y Gunset (2009) han explorado las interacciones verbales que se producen entre docentes y alumnos del nivel inicial, caracterizados como provenientes de contextos desfavorecidos.

\section{b. Usos de conocimientos extra-escolares en el ámbito del aula}

Mientras que en el anterior grupo los estudios realizan predominantemente una comparación entre aspectos de los aprendizajes escolares y extra-escolares, en este grupo de trabajos el foco está puesto en la escuela y se indagan los usos en el aula de conocimientos y formas de aprender extra-escolares. Las investigaciones desde la psicología cognitiva sobre las ideas previas del alumnado son el ejemplo tradicional de este tipo de abordajes (Limón y Carretero, 1997) y han llamado la atención sobre la importancia de atender en el aula a las ideas previas que provienen de la experiencia cotidiana del alumnado y que son eficaces para predecir determinados comportamientos pero que no siempre coinciden con el punto de vista escolar y/o científico.

En el campo de la psicología discursiva un estudio de Candela (2006) en América Latina analiza también las interacciones discursivas generadas entre docentes y alumnos durante las clases de ciencias, considerando cómo son utilizados los conocimientos extra-escolares de los niños, y en particular, aquellos asociados a las 'evidencias empíricas', es decir, “...lo que es descrito por los participantes como lo que 'se ve' u 'observa en la realidad'...” (Candela, 
2006:806). La autora parte del supuesto de que la experiencia extra-escolar de los alumnos constituye uno de los aspectos clave para movilizar y construir el conocimiento que en la escuela es establecido como científico.

Los denominados enfoques socioculturales han resultado un marco fructífero para estas indagaciones. Por ejemplo, Moll y colaboradores (1990, 2005) indagaron los 'fondos de conocimientos' familiares y comunitarios en una comunidad de clase trabajadora y principalmente mexicana en Arizona, EE. UU., con el objeto de mejorar las prácticas de enseñanzaaprendizaje en el contexto áulico. Si bien el concepto de fondos de conocimientos será desarrollado más adelante, cabe señalar aquí que apunta a la descripción de los conocimientos disponibles en los contextos familiares de alumnos pertenecientes a una determinada comunidad, con el fin de recuperar, en espacios escolares, saberes producidos y transmitidos en distintos contextos comunitarios (Moll y Greenberg, 1990). En otras producciones, Davis (2007) y Black (2007) desarrollan el concepto de modelos culturales y los combinan con la teoría de la actividad para investigar cómo los discursos de los niños acerca de la lectura (especialmente aquellos discursos construidos por fuera del aula) median las prácticas de enseñanzaaprendizaje en el aula.

\section{c. Aprendizaje en contextos extra-escolares de ciertos "contenidos escolares"}

En un tercer grupo, en cambio, el énfasis está puesto en los espacios extra-escolares, y el interés es el análisis de la apropiación de contenidos tradicionalmente considerados 'escolares' en esos otros espacios. A pesar del foco en ambientes extra-escolares, sigue en estos casos existiendo un interés por informar, o desafiar, a las prácticas pedagógicas escolares. En este grupo se encuentran todas las investigaciones que han atendido al aprendizaje de la lectoescritura y de ciertos contenidos matemáticos en contextos extra-escolares, tanto para identificar los precursores de estos aprendizajes, como para analizar las formas alternativas o complementarias de aprender los mismos en contextos no formalizados. Desde una tradición piagetiana, y recurriendo al método clínico como estrategia de indagación, se destacan la clásica investigación de Carraher et al (1998) en Brasil sobre las estrategias diferenciales de cálculo de niños brasileños en situaciones de trabajo en una feria y resolviendo problemas de cálculo escolares; y las indagaciones iniciales que Ferreiro y colaboradores realizaran en la Argentina sobre los usos de las matemáticas en contextos inflacionarios y en sus desarrollos tempranos sobre adquisición de la lectoescritura (Ferreiro, 1986; Ferreiro y Teberosky, 1979). Estas in- 
vestigaciones clásicas resultan sumamente relevantes porque muestran un claro interés por pensar o desafiar la educación formal, privilegiando como ámbito de indagación a los ambientes extra-escolares.

Otro ejemplo más reciente es el estudio de Andrews y Yee (2006) en Inglaterra, que reporta sobre las experiencias cotidianas de niños y niñas pertenecientes a minorías étnicas en relación a la lectoescritura y matemáticas, identificando variadas prácticas cotidianas de producción de conocimientos y contrastándolas con la perspectiva de los docentes sobre el desempeño escolar de esos/as alumnos/as.

Existen estudios en México que, desde una perspectiva antropológica, amplían la mirada para reflexionar respecto de los procesos de aprendizaje de la lectoescritura en diferentes ámbitos. Uno de ellos es el de Rockwell (1992) que analiza las relaciones que se establecen entre la oralidad y la escritura en las prácticas narrativas utilizadas en una zona rural, destacando cómo la escritura adquiere un significado en el marco de interacciones orales, a la vez que se asume como recurso relevante en las instancias de negociación y confrontación. Por su parte, a partir de un trabajo empírico que si bien es con adultos resulta de relevancia para nuestra indagación, Kalman (2003) desarrolla una reflexión teórica desde una perspectiva sociocultural, con el objeto de examinar el acceso a la escritura considerándola como un proceso social en el que la participación de los individuos en situaciones de interacción con otros es condición necesaria para su aprendizaje. Es interesante la distinción que realiza la autora entre la noción de 'disponibilidad' de la cultura escrita, que alude a las condiciones materiales que permiten la práctica de la lectura y la escritura, y la noción de 'acceso' a la cultura escrita, que refiere a las condiciones sociales requeridas.

\section{d. Espacios de mediación escuela-familia}

El análisis de los espacios de interacción entre la escuela y las familias resulta otro foco elegido en estas discusiones en tanto se entiende que son instancias en que las diferentes experiencias de aprendizaje y de sentidos se ponen en diálogo o tensión. Con respecto a los vínculos entre las escuelas y las familias, se observan líneas de trabajo que focalizan su atención en el potencial que tiene la participación activa de las familias en lo que respecta al logro de experiencias escolares exitosas de sus niños (Elichiry, 2007; Ada, 2003; Epstein y Dauber, 1991). 
Hughes (2006a, 2006b) ha desarrollado en el Reino Unido una investigación sobre los procesos de intercambio y transformación de conocimiento entre familias y escuelas recurriendo también al concepto de fondos de conocimientos desarrollado por Moll y Greenberg. En la Argentina, utilizando también aspectos de los enfoques socio-culturales, se encuentra el programa de investigaciones del equipo de Elichiry y colaboradores sobre diferentes espacios de mediación entre el contexto escolar y familiar, tales como los espacios tutoriales, los deberes escolares y el lugar de los medios masivos de comunicación (Elichiry, 2007; Elichiry et al, 2005 y 2008).

Desde una perspectiva antropológica, el trabajo de Sagastizabal (2000) en una comunidad de origen toba, se ha focalizado en el impacto que, sobre la identidad escolar del alumnado, pueden tener las diferencias de interpretación entre la escuela y la familia acerca del rendimiento de los niños.

En el análisis de experiencias, vale mencionar el caso estudiado por Jasis y OrdoñezJasis (2004) en EE.UU. sobre el proyecto 'La Familia', donde un grupo de padres de alumnos que asistían a una escuela ubicada en la Bahía de San Francisco -la mayoría de ellos inmigrantes- se unieron con el fin de mejorar la situación escolar de sus hijos, habitualmente identificados con bajos rendimientos. Esta experiencia fue expandiéndose a otras cinco instituciones de la zona, generando en todas ellas cambios considerados altamente positivos por la comunidad, tanto en términos de logros académicos como también de los vínculos establecidos entre las familias y las escuelas. Uno de los aspectos a destacar es que, como parte de los resultados de la experiencia, los alumnos de origen latinoamericano y sus familias comenzaron a hacerse más 'visibles' y orgullosos respecto de sus aportes en la institución escolar, dando como resultado una evidente mejora en el rendimiento académico, como así también en lo que respecta a su autoestima y motivación para tomar iniciativas.

A modo de cierre del presente apartado, cabe hacer mención a los tipos de diseño de los estudios abordados hasta aquí. Con la excepción de los estudios realizados en el marco de la psicología cognitiva, cuyas investigaciones han incluido también diseños con elementos de corte cuasi-experimental, el resto de las investigaciones mencionadas se caracterizan por tratarse de abordajes cualitativos, con diseños que incluyen principalmente etnografías y/o observaciones participantes, estudios de caso, y el uso del método clínico en los abordajes de 
corte piagetiano. Llamativamente, es común encontrar en estas investigaciones algunos elementos de la investigación-acción y de la investigación participativa, con el objeto de incluir en el proceso de producción las perspectivas de los mismos docentes, alumnos y familiares sobre las potencialidades y límites de articulación de los aprendizajes cotidianos con los escolares. El problema de las relaciones de las prácticas escolares con los aprendizajes que se dan en otros contextos parece ser un espacio que invita a la construcción colectiva del conocimiento por parte de los mismos actores. A modo de ejemplo, vale mencionar la experiencia "El puente" relatada por González y Moll (2002) en una comunidad latina, donde los docentes realizaron trabajos etnográficos en las comunidades de sus alumnos y utilizaron estas experiencias para redefinir la pedagogía del aula.

\section{Fundamentaciones para la investigación empírica sobre el problema ${ }^{3}$}

En líneas generales, dos tipos de argumentos son utilizados en las investigaciones para justificar la legitimidad de analizar contextos de aprendizaje y formas de aprender extraescolares partiendo de un interés pedagógico o escolar. Estos refieren a la inclusión en la escuela de conocimientos y aprendizajes extra-escolares como a) generadores de motivación y promotores de aprendizajes significativos en el alumnado, y b) como una forma de justicia social.

Con respecto al primer argumento, una de las afirmaciones corrientemente sostenidas es que la inclusión en la escuela de conocimientos y aprendizajes de la vida cotidiana de los alumnos contribuye a desarrollar un curriculum más atractivo para los alumnos, a lograr un mayor interés y compromiso en la participación, así como a mejorar el rendimiento escolar (Lacasa et al 1996; Zipin, 2009; González y Moll, 2002; Marsh, 1999). Un claro ejemplo es la investigación de Buckingham y Sefton-Green, 1994 (mencionada por Marsh, 1999), que demostró que la inclusión en el aula de escuelas secundarias de lecturas y escrituras relacionadas a producciones de moda en los medios de comunicación tenía un efecto poderoso en la mejora de los niveles de motivación de los jóvenes.

\footnotetext{
${ }^{3}$ Una versión preliminar de esta sección fue presentada en el V Congreso Nacional y III Internacional de Investigación Educativa. Ponencia "El aprendizaje en diferentes contextos. La producción académica sobre las articulaciones posibles entre el aprendizaje escolar y en otros ámbitos”. Universidad Nacional del Comahue. 21 a 23 Octubre 2009. Autoras: Silvina Cimolai y Julia Lucas.
} 
Este argumento es alternadamente un supuesto de partida, que justifica el estudio, o una conclusión, resultado de análisis cualitativos de las experiencias analizadas. Solamente en el caso de la ya mencionada investigación de Hughes, este aspecto fue específicamente un objetivo de investigación, en tanto el proyecto se proponía "desarrollar, entender, medir, evaluar y difundir formas en que el rendimiento escolar y la disposición a aprender de los alumnos puede ser fortalecida por un proceso de intercambios y transformaciones de conocimientos entre los padres, los alumnos y los docentes" (Hughes, 2006a:2). Los resultados, no obstante, no pudieron ser concluyentes con respecto a la mejora en el rendimiento y la motivación, si bien argumentaron posibles efectos de otras variables que no habían sido consideradas en el diseño.

En una misma línea argumentativa, se menciona que la exploración de lo extra-escolar tiene un impacto directo en los mismos alumnos, en tanto amplía sus imágenes o identidades como 'aprendices' al re-significar modos de aprender en los que participan 'exitosamente' en la vida cotidiana. También se advierte un impacto en las representaciones que los docentes construyen acerca de qué es aprender para sus alumnos y los modos en que los mismos participan activamente de situaciones de aprendizaje por fuera del aula. Andrews y Yee (2006), en su investigación sobre fondos de conocimiento familiares en relación a las matemáticas, señalaron que la indagación de formas de aprender de los alumnos en otros contextos impactó en las mismas representaciones que los docentes tenían acerca de las capacidades de esos mismos alumnos en el ámbito del aula.

Con respecto a la segunda fundamentación mencionada, como forma de justicia social, es importante señalar cómo la mayor parte de estas investigaciones señala explícitamente un interés por la problemática del fracaso escolar, las desigualdades educativas y el rendimiento diferencial que los sectores populares y las minorías étnicas presentan en las escuelas. Significativamente, las poblaciones analizadas suelen pertenecer, en su extensa mayoría, a sectores desfavorecidos o 'minorías'.

En un estudio sobre las experiencias de alfabetización preescolar en niños pertenecientes a familias desfavorecidas, Anderson et al (2002:269-270) señalan: "Generalmente se cree que las experiencias en el hogar de niños de bajos recursos y de 'minorías' étnicas no los preparan eficazmente para aprender a leer y escribir. Los antecedentes familiares de dichos niños se citan a menudo como una fuente de sus dificultades escolares en la lectura y la escritura, y 
se asume que, en lo que corresponde a éstas, existe disparidad entre el hogar y la escuela. Los estudios en gran escala son de poca ayuda en la cuestión de la disparidad (...). Al investigar los ambientes de alfabetización de los niños (...), esperamos poder arrojar luz sobre las experiencias preescolares de los niños y, por consiguiente, proporcionar información que las escuelas y los maestros puedan utilizar para ayudarles a responder de forma más eficaz a los niños de bajos recursos y de "minorías"”.

En la Argentina, cabe mencionar la fundamentación de Elichiry et al (2005) en el subproyecto de investigación sobre deberes escolares, basándose en la necesidad de analizar los espacios de mediación e interacción entre la escuela y la familia atendiendo a los micro procesos cotidianos que conducen a la exclusión escolar y a los aspectos que, desde la escuela, contribuyen a estructurar las desigualdades educativas.

Otro aspecto que atraviesa a estas investigaciones es que en el diseño y análisis de la información, las mismas se posicionan políticamente discutiendo la afirmación de que entornos familiares y sociales 'deficitarios' serían la causa de las dificultades de rendimiento escolar de niños y niñas pertenecientes a sectores desfavorecidos. Asimismo, buscan mostrar las variedad de recursos, intercambios y conocimientos con los que estos alumnos y alumnas interaccionan exitosamente en el contexto cotidiano (ver las descripciones de Carraher et al, 1998; Moll y Greenberg, 1990; Andrews y Yee, 2006; Borzone y Rosemberg, 2000). Por ejemplo, Moll, Amanti, Neff y González (2005) buscan captar la variedad y riqueza de los recursos culturales e intelectuales que se ponen en juego en los hogares con el objeto de incluirlos en las prácticas escolares. Explicitan la importancia de este tipo de abordajes cuando se trata de una comunidad que tradicionalmente ha sido analizada como pobre en términos no sólo económicos sino también de la calidad de las experiencias en las que los niños y niñas participan.

En la misma línea, las indagaciones ya mencionadas de Emilia Ferreiro y colaboradores en la Argentina sobre los usos de las matemáticas en contextos inflacionarios (Ferreiro, 1986) y sus desarrollos tempranos sobre adquisición de la lectoescritura (Ferreiro y Teberosky, 1979), llevan también implícitos un interés educativo y un posicionamiento político con respecto al fracaso escolar de los sectores desfavorecidos como un fracaso de la institución escolar, identificando que los niños de estos sectores interaccionan eficazmente en su vida cotidiana con objetos culturales con los que en la escuela fracasan. Ferreiro y colaboradores 
consideraron a "estos contenidos como objetos culturales antes que escolares, con una presuposición fuerte hacia la desfuncionalización escolar en relación a estos objetos" (Caruso y Fairstein, 1997:191, cursivas en el original). Se trataba en primer lugar de un reconocimiento al carácter lógico que tiene el pensamiento infantil cotidiano, cuestionando al mismo tiempo los sinsentidos que se producen en el aprendizaje escolar, como en el caso del cálculo, donde el uso de estrategias formales de cálculo en lápiz y papel descontextualiza la operación y lleva a producir respuestas ilógicas (Caruso y Fairstein, 1997; Carraher et al, 1998).

Partiendo de las afirmaciones de que el curriculum escolar se organiza en función del capital cultural encarnado en las "posiciones socio-estructurales de elite”, Zipin (2009) enumera los efectos de diseñar un curriculum escolar en torno a los conocimientos que tienen valor de uso en las vidas cotidianas de los aprendices de cualquier sector social. Concordando con Moll y González, 1997 (citado por Zipin, 2009) sostiene que transformar la diversidad de los estudiantes en recursos pedagógicos implica cuestionar la tradicional visión de las experiencias de los niños de sectores desfavorecidos como escenarios desde los cuales los chicos deben ser 'salvados' o 'rescatados', en tanto, al igual que cualquier entorno comunitario, contienen conocimientos y experiencias de valor para promover el desarrollo educacional, y no sólo 'problemas'.

En líneas generales estas posiciones sostienen que, dados los códigos culturales de elite que dominan los estándares de la educación en general, y en orden de hacer justicia con respecto a los que están en posiciones de mayor debilidad en las relaciones sociales de poder, es necesario redistribuir esos códigos de elite haciéndolos explícitos y practicables. Y esos esfuerzos de redistribución necesitan andamiarse de un curriculum que priorice el reconocimiento, el valor y el uso del conocimiento de la vida familiar y comunitaria de los aprendices. Este esfuerzo de redistribución es crucial para facilitar el éxito académico, pero también es crucial en términos éticos para mantener 'vivas' las diferencias, $\mathrm{y}$, en términos pragmáticos, para promover y sostener el interés y participación de los alumnos.

\section{Los objetos culturales explorados}

Seguramente debido al hecho de que, en general, las investigaciones que nos ocupan se han originado en un interés por mejorar las prácticas escolares, en el análisis de los aprendizajes en contextos extra-escolares, los contenidos u objetos culturales que se indagan apare- 
cen recurrentemente definidos en función de la clasificación disciplinar propuesta por el curriculum escolar. Dentro de estos contenidos, las indagaciones relacionadas a la lectoescritura (Hughes, 2006a; Anderson et al, 2002; Ferreiro y Teberosky, 1979; Lacasa et al, 1996, Davis, 2007 y Black, 2007) y a las matemáticas (Carraher et al, 1998; Hughes, 2006a; Ferreiro, 1986) son las predominantes, observándose también una presencia -aunque menor- de contenidos de ciencias y física, específicamente en la tradición de indagación ya mencionada sobre ideas previas de los alumnos (Limón y Carretero, 1997). Esto señala una tendencia que sesga el análisis del aprendizaje extra-escolar, resultando por momentos un problema metodológico para los diseños, por ejemplo debido al hecho argumentado en algunas de estas investigaciones de que algunos aprendizajes en el hogar que implican matemáticas o lectoescritura, no son considerados como tales por los actores (Hughes, 2006a). La identificación disciplinar que las investigaciones realizan con respecto al aprendizaje de ciertas actividades de lectura, escritura o cálculo en el ámbito cotidiano, presenta algunos problemas que no son siempre considerados cuando se compara dicho ámbito con el aprendizaje escolar. Esto afecta el tipo de discontinuidades que se detectan, algunas de las cuales se desarrollarán en el siguiente apartado. Si bien el dominio en juego es similar, hay una diferencia entre el conocimiento disciplinar y las formas en que el dominio se produce en la vida cotidiana y en la escuela. Por ejemplo, siguiendo a Carraher et al (1998), las matemáticas como disciplina conformarían un conocimiento formal, con una lógica deductiva de producción de conocimiento, mientras que las matemáticas, como actividad humana, resultarían una forma particular, efectiva y económica de organizar ciertos acontecimientos del mundo permitiendo establecer relaciones entre los objetos, como así también cuantificarlos, sumarlos, medirlos, restarlos, dividirlos. Como actividad humana, las matemáticas no siguen las leyes de construcción lógica y deductiva del conocimiento disciplinar y suelen no ser aceptadas como válidas en el contexto formal. No obstante, ciertas formas de cálculo cotidianas llevan a resultados aceptables en el contexto cotidiano, en la medida en que, de hecho, resuelven problemas (Carraher et al, 1998).

Con respecto al aprendizaje de la lectura y la escritura, los diseños que han buscado analizar diversos ámbitos de producción como los hogares, las escuelas, los lugares de trabajo, las organizaciones sociales y la comunidad, comparten -aún desde diferentes perspectivas teóricas- que el aprendizaje de la lectoescritura es algo más que la adquisición de sus aspectos rudimentarios o mecánicos, promoviendo así la reconceptualización de la lengua oral o escrita como una práctica social y contextualizada. Tal como señala Freebody (mencionado en Kalman, 2008) para el caso de la cultura escrita, estos trabajos han contribuido a la investigación 
a través de la documentación de cómo se logra y se valora la escritura y cómo se inserta en sus comunidades y sus lenguajes.

No obstante, son quizás las investigaciones sobre fondos de conocimiento las que han innovado en esta tendencia y por eso merecen un desarrollo más detenido. El concepto de fondos de conocimientos se utilizó por primera vez para describir los conocimientos y saberes disponibles en los ambientes familiares de alumnos pertenecientes a una comunidad principalmente mexicana en USA (Moll y Greenberg, 1990) y ha sido luego retomado en una variedad de investigaciones que comparten el interés por recuperar, en la escuela, los conocimientos de la vida extra-escolar del alumnado proveniente de grupos desfavorecidos o minorías étnicas (Andrews y Yee, 2006; Martin-Jones y Saxena, 2003; Hughes, 2006a; Zipin, 2009). En línea con los trabajos vigotskianos, este concepto otorga especial relevancia a las actividades productivas y a los contextos socioculturales en las que las mismas se desarrollan, entendiendo que los fondos de conocimiento no son posesiones de los integrantes de las familias, sino que se hacen manifiestos a través de hechos y actividades, en tanto son "características de la 'gente que realiza una actividad"' (Moll y Greenberg, 1990:379). Según estos autores, estos fondos conforman "un manual de funcionamiento de la información y las estrategias esenciales que necesitan los hogares para mantener su bienestar" (Greenberg, 1989:2). Esto significa que las familias y comunidades constituyen redes en las que los conocimientos no son posesiones individuales, sino interaccionales, puesto que se requiere la realización de actividades conjuntas o complementarias con los demás integrantes. La noción de 'confianza' se torna aquí fundamental en el marco del intercambio recíproco generado en las 'redes sociales' que se conforman entre las familias al generar contextos propensos a la transmisión y socialización de saberes y habilidades (Moll y Greenberg, 1990).

Cabe destacar que el concepto de fondos de conocimiento, aún cuando se refiere a las actividades que los niños realizan en contextos familiares y comunitarios, es utilizado en todas estas investigaciones con un interés educativo y escolar. El objeto de recuperar esos fondos de conocimientos está siempre relacionado al interés de mejorar las prácticas escolares creando sentidos más acordes a la vida diaria de estos niños. Partiendo de una perspectiva vigotskiana, los conocimientos familiares no se indagan en función de su pertenencia disciplinar, sino otorgando especial relevancia a las actividades productivas y a los contextos socioculturales en los que participan los sujetos. Parten, además, de una necesidad teórica de articu- 
lar la labor productiva con las prácticas de aprendizaje, o bien, las actividades laborales con la enseñanza escolar (Moll y Greenberg, 1990).

Continuidades y discontinuidades entre aprendizaje escolar y extra-escolar

Los estudios comparativos de situaciones de enseñanza-aprendizaje en contextos cotidianos y escolares suelen privilegiar una estrategia de análisis centrada en la identificación de las discontinuidades entre ambos contextos. No obstante, tanto nuestras indagaciones, como algunas investigaciones encontradas, cuestionan, por un lado, la homogeneidad con que se definen las situaciones de aprendizaje dentro de cada contexto, mientras que, por otro lado, muestran las continuidades o interacciones que suelen existir entre los contextos, por ejemplo, identificando la existencia de prácticas y situaciones extra-escolares que alteran o transgreden las lógicas escolares, o la observación de lógicas de aprendizaje típicas del contexto escolar, operando en algunos contextos cotidianos.

A continuación, se presentan las caracterizaciones de tres grandes tipos de discontinuidades señaladas generalmente, para luego exponer algunos aportes que cuestionan aspectos de estas distinciones o hacen énfasis en las continuidades entre contextos. Nuestro argumento es que, si bien las identificaciones de las diferencias entre las formas de aprender en la escuela con respecto a otros contextos resulta un problema de investigación relevante, las formas en que tradicionalmente se ha priorizado su presentación tienden, en algunos casos, a estereotipar, simplificar y homogenizar contextos de mayor complejidad.

Discontinuidad 1: direccionalidad, participación restringida vs. multidireccionalidad, participación variada

Un primer aspecto que recurrentemente se menciona en las investigaciones es el alto grado de unidireccionalidad que tienen las interacciones e intercambios verbales en el contexto escolar, a diferencia de lo que sucede en algunos otros contextos de aprendizaje (Cazden, 1991; Borzone y Rosemberg, 2000; Prado, Canigia y Gunset, 2009; Kalman, 2003; Wells, 1986).

Así, por ejemplo, en la provincia de Tucumán (Argentina), Prado, Canigia y Gunset (2009) al analizar las características que adquieren las interacciones verbales entre docentes y 
alumnos en el contexto escolar, señalan que las formas en que el docente regula el diálogo en la clase inhabilita y desautoriza ciertos usos de la palabra en los niños. Es interesante notar que aún cuando los docentes ponían en relación las características socio-económicas de los niños y/o sus familias con el aprendizaje del lenguaje, se advertía un alto grado de direccionalidad por parte del docente en las interacciones. Tal como señalan Prado, Canigia y Gunset, mientras los docentes enuncian la necesidad de trabajar "con lo que el niño trae de su casa solamente", la práctica muestra movimientos que contradicen tales afirmaciones.

En esta misma línea, la investigación realizada por Borzone y Rosemberg (2000) en el norte argentino señala que las interacciones verbales que los niños kollas tienen con pares y adultos en el contexto de su hogar difieren considerablemente de aquéllas que se presentan en el marco del contexto escolar, donde los derechos de participación de los niños y su responsabilidad en las conversaciones se encuentran restringidos por el control del maestro. Para estas investigadoras, la direccionalidad se hace evidente a través de la constatación de que son los docentes quienes hablan la mayor parte del tiempo, dando inicio a los intercambios con mayor frecuencia, instando a la participación de los alumnos a través de la formulación de preguntas cerradas, limitando la intervención de los niños a respuestas breves o frases elípticas. Generalmente, cuando los niños inician los intercambios, es sólo para solicitar la aprobación sobre la actividad que se está realizando o para solicitar alguna indicación al respecto.

Desde una perspectiva sociolingüística, Wells (1986) argumenta que las características que tiñen las interacciones entre maestros y alumnos se orientan a delimitar las diferencias de poder entre la posición del maestro respecto a la del niño. En este sentido señala que "La función social oculta de las preguntas que piden una demostración de esas aptitudes [...] marca así la diferencia de status entre maestro y alumnos y se conserva el control de su atención y participación” (Wells, 1986:101).

Por su parte, Borzone y Rosemberg señalan que esta forma de interacción, no sólo estaría limitando la posibilidad de interacción verbal por parte de los niños, sino que además deviene en un formato que, basado en preguntas cerradas y respuestas esperadas, no promueve el tipo de actividad cognitiva necesaria para el desarrollo del aprendizaje. A diferencia de lo que sucede en la escuela, los niños poseen mayores oportunidades para hablar en sus hogares, lo que produce una mayor posibilidad de aprendizaje lingüístico y cognitivo. 
Las oportunidades de interactuar, según las investigaciones citadas hasta aquí, varían de acuerdo al contexto, y muestran que los niños hablan mucho menos en la escuela que en el hogar. Tal como muestra Wells (1986) la cantidad de palabras dirigidas de niño a adulto, así como la longitud de las secuencias interaccionales registradas en el hogar superan a aquellas producidas en el marco escolar, mostrando de esta manera que en el hogar los niños suelen tomar parte en secuencias más largas de interacción a la vez que menos jerarquizadas que en la escuela. Otra indicación de oportunidad en la conversación puede visualizarse en la complejidad sintáctica puesta en juego en cada uno de los contextos. La del niño es mucho más baja en la escuela, mientras que la del adulto muestra una tendencia en sentido opuesto, es decir, que el niño explota menos sus recursos cuando habla con su maestro que cuando habla con sus padres. En la escuela los niños inician menos conversaciones y hacen una menor cantidad de preguntas que en el hogar. El docente es concebido aquí como un mediador entre los alumnos y los conocimientos en dos sentidos, en tanto, por un lado, es él quien interpreta de un determinado modo los contenidos a enseñar, mientras que por otro lado, es también quien 'representa', frente a los alumnos, la autoridad de dichos contenidos (Edwards, 1995).

Moll et al (2005) señalan que una característica central que define a las interacciones cotidianas y que se diferencia de los intercambios en el aula es su reciprocidad. Afirman que la forma de los intercambios en el ámbito familiar o comunitario genera prácticas basadas en la reciprocidad y la confianza, y lleva al desarrollo de redes de intercambio y aprendizaje a largo plazo. Asimismo, las redes y las esferas de aprendizaje en las familias de los niños y niñas son flexibles, adaptativas y activas, e involucran a muchas personas, generando, incluso, diferentes formas de relación con una misma persona. Moll et al (2005) definen a estas redes como densas y con múltiples filamentos mientras que señala que la relación en el aula es delgada y no-múltiple, y que el conocimiento del niño por parte del docente es acotado, limitándose a su desenvolvimiento en el contexto del aula.

\section{Discontinuidad 2: Sentido impuesto y desconocido vs sentido compartido}

Conocer o intuir el sentido o propósito de las actividades realizadas por parte de los niños es otra de las discontinuidades encontradas en los trabajos aquí presentados. Así por ejemplo, Wells (1986), sostiene que en el cotidiano del hogar niños y niñas hablan o aprenden, en gran medida, a partir de la necesidad de dar respuesta a sus experiencias. Los adultos que los rodean, suelen acompañar las búsquedas o facilitar las respuestas a las inquietudes 
infantiles. En este contexto y en relación al papel que desempeñan los padres en las actividades que comparten con sus hijos en el hogar Wells (1986) señala "la principal motivación para aprender procede de dentro, y apenas hay un esfuerzo sistemático por darle instrucción, salvo en cuestiones tales como la seguridad y una conducta aceptable a nivel social" (Wells, 1986:86). Asimismo, se menciona recurrentemente que en las actividades en el hogar, por ejemplo, los niños interaccionan con objetos como la lectoescritura en contextos que resultan significativos y con sentido, a diferencia de lo que ocurre en la escuela con actividades similares (Hughes, 2006a). En este sentido Gumperz (1984) señala que en esos ámbitos los eventos comunicativos no tienen lugar en espacios vacíos de significados culturales y sociales: cada hablante, escritor/lector, aporta su propia visión del mundo, prácticas de lenguaje, historia y experiencia con los participantes a un evento comunicativo determinado. A diferencia de lo que sucede en el hogar, se señala que en la escuela, generalmente, no se explicita el propósito de la actividad diseñada para el aprendizaje, y que, mediante las características que adquieren las interacciones en el contexto escolar, se condiciona la posibilidad de discutir el significado respecto de aquello que se enseña.

Con respecto a la práctica de aprendizaje en sí misma, se señala un status diferente en la definición de las actividades escolares y extra-escolares. En primer lugar, se describe que lo realizado dentro de la escuela es fácilmente reconocido por los actores como aprendizajes, mientras que resulta más difícil para los actores identificar como aprendizajes aquellos que se llevan a cabo en su vida cotidiana (Nakache, 2007). Esto pareciera deberse al hecho de que mientras en las escuelas el aprendizaje es el motivo central de la actividad escolar, en las situaciones extra-escolares los niños y niñas participan de actividades donde el aprendizaje no es, necesariamente, el motivo central, sino el resultado de la participación en las mismas (Moll et al, 2005; Hughes, 2006a). No obstante, se señalan complejidades como las analizadas por Moll y Greenberg (1990), mostrando que en ciertas actividades familiares la apropiación de habilidades y valores por parte de los niños es un objetivo explícito de los miembros más experimentados, aunque para ello se valen de la inclusión de los niños en tareas del hogar que no son diseñadas específicamente para ese aprendizaje. 
Discontinuidad 3: Aislamiento de las actividades vs aprendizaje por participación en las actividades

A diferencia de lo que sucede en el hogar, las aulas son analizadas como aisladas de la vida social y de los recursos disponibles en la comunidad, señalando por ejemplo que son muy escasas las veces en que los docentes basan sus prácticas en los recursos de los fondos de conocimiento con que los alumnos interaccionan por fuera de la escuela (Hughes, 2006a; Moll y Greenberg, 1990). En cambio, en el hogar, a diferencia de la escuela, los procesos de enseñanza y aprendizaje se caracterizan por no separar explícitamente a los niños de las actividades realizadas por los adultos. "Los niños aprenden observando, curioseando en sus desplazamientos por la comunidad, incorporándose y participando en las tareas cotidianas y recibiendo ayuda de los adultos o compañeros más competentes a través de demostraciones, iniciaciones verbales y señalamientos no verbales" (Borzone y Rosemberg, 2000:63). Desde edades tempranas los niños aprenden observando las tareas realizadas por los miembros expertos de su comunidad. La exploración forma parte también del conjunto de operaciones realizadas por los niños en ámbitos como el hogar o los espacios comunitarios.

Según Borzone y Rosemberg (2000) en la escuela, los niños aprenderían cuestiones relacionadas con la lectura, la escritura y el cálculo como objetos de aprendizaje en sí mismos esto es, el aprendizaje de letras o sílabas, o el algoritmo, por ejemplo- desvinculándose de los usos sociales, culturales o prácticos que pueden tener en la vida cotidiana. La mayor parte de aquello que se trasmite se realiza de forma verbal y son escasas las veces en que se interpela a los alumnos a la producción de material escrito. No obstante, la investigación muestra la forma en que, desde los inicios de la escolarización, niños y niñas adquieren claros conocimientos sobre las normas sociales que organizan la experiencia escolar. "Aprenden a hablar dentro de la estructura de participación que crea el docente: el niño responde a las preguntas del docente y sólo inicia durante la realización de la tarea para pedir la evaluación del maestro" (Borzone y Rosemberg, 2000;61).

\section{Interacciones o continuidades}

Diferentes trabajos muestran que los aprendizajes caracterizados a partir de una división estricta entre contextos no permiten captar los matices que pueden presentarse entre los mismos. Así, por ejemplo, se señala la existencia de situaciones de aprendizaje o intercam- 
bio/interacción que ponen en cuestión la caracterización homogénea de las formas de aprender en el contexto escolar. Por otra parte, otros trabajos señalan la existencia de situaciones en el hogar -como las actividades de enseñanza explícita de la lecto-escritura por parte de los adultos del hogar -que estarían mostrando la irrupción de formas o métodos escolares en los contextos del hogar.

Gandulfo (2007) apela al concepto de ideología lingüística, en tanto ella permite hablar de la relación entre las formas de habla, en este caso, los usos de una determinada lengua en determinada situación, las ideas acerca de por qué debieran realizarse esos usos y no otros, y la estructura social. La introducción de esta categoría, según Gandulfo, permitiría comprender el significado que estas interacciones adquieren para los hablantes de una u otra lengua. En este sentido señala que “...los niños aprenden no sólo a hablar, sino -y a la vezdónde, cómo, y con quién, es decir, aprenden a comportarse en la comunidad de la cual son parte" (Gandulfo, 2007:68). No obstante, la autora muestra instancias donde se produce una alteración -que puede ser leída, quizá, en términos de resistencia- donde los hablantes transgreden estos aprendizajes y rompen estos formatos. Algunos ejemplos se presentan cuando en algunos ámbitos de la escuela se producen conversaciones en idioma guaraní que excluyen a los docentes, o cuando los padres afirman no enseñar a sus hijos el guaraní para evitar procesos de exclusión, pero sí reconocen hablarlo entre adultos, provocando que los chicos aprendan de esos intercambios. Tal como señala Gandulfo (2007), la ideología generaliza mientras que las prácticas muestran los matices que se pueden presentar.

Por su parte Lacasa et al (1996) señalan que cuando en las familias se organizan actividades cuyo objetivo es el aprendizaje de la lecto-escritura, generalmente se apela a métodos que son más propios de la escuela y que tienen poca relación con las actividades cotidianas, dado que el aprendizaje de la lectoescritura es concebido por las familias como una actividad eminentemente escolar. La instrucción verbal explícita (o instrucción directa) suele aparecer en los contextos del hogar cuando los adultos desean transmitir ciertas convenciones sociales o, cuando los niños mayores de la familia desean enseñar a los más pequeños contenidos escolares como números, letras, o colores. En estos casos, se suele recurrir a métodos de enseñanza que son empleados en la escuela. De este modo "La adquisición de conocimientos tiene lugar en el contexto de la actividad conjunta y la colaboración a través de formas implícitas y explícitas de enseñanza mencionadas. Los adultos estructuran la actividad adaptando sus requerimientos al nivel de habilidades del niño. El niño conoce el objetivo general de la tarea y 
se incorpora a ella en un principio para la realización de submetas" (Borzone y Rosemberg, 2000:66-67).

El trabajo de Achilli (1996), también centrado en las percepciones de los distintos actores involucrados en la cotidianeidad de las escuelas, focaliza su atención en el vínculo niñoescuela en contextos de pobreza urbana y 'diversidad sociocultural'. La autora se refiere, desde una perspectiva crítica, a los estudios tradicionales realizados en el campo de la antropología (su disciplina de origen) y la sociología, dado que han 'dicotomizado' los vínculos entre las escuelas y las familias sin tener en cuenta los aspectos históricos, las particularidades y las relaciones mutuas existentes. A diferencia de este tipo de concepciones, más bien estereotipantes de los contextos intervinientes, Achilli propone un análisis relacional de lo que identifica como 'experiencias formativas' del niño (familiar y escolar), es decir, el “...conjunto de prácticas y relaciones cotidianas en las que se involucra el niño al interior de particulares modalidades institucionales -escolar, familiar- que van condicionando el carácter y el sentido que adquiere para otros y para él mismo las apropiaciones -cognitivas y normativas- que realiza" (Achilli, 1996:40).

\section{Conclusiones y recomendaciones}

Un primer aspecto a destacar, cuya reflexión se alimenta del trabajo empírico que venimos desarrollando en instituciones educativas del Gran Buenos Aires (Argentina), es que aún reconociendo la profunda complejidad y las variaciones en las investigaciones abordadas (algunas de las cuales no han sido desarrolladas en esta presentación), cuando las mismas identifican aspectos diferenciales del aprendizaje escolar y de otros contextos de aprendizaje, la línea divisoria de las discontinuidades que se señalan entre unos y otros se presentan por momentos como borrosas y cuestionables (como en el caso de la afirmación de la no articulación entre contextos, o sobre la pasividad-actividad que pareciera tener el niño o niña en un contexto escolar con respecto a un contexto de aprendizaje familiar). Si bien estos trabajos se posicionan en demostrar cómo los niños y niñas aprenden formas de participación diferencial en cada contexto de las actividades, también es necesario reconocer instancias donde se produce una alteración -que puede ser leída quizá en términos de resistencia- donde los niños transgreden las lógicas específicas de cada contexto, y éstas, a su vez, se superponen y recrean conjuntamente. En este sentido, ante la dualidad o el carácter dicotómico con que suelen caracterizarse contextos escolares y no escolares, así como los aprendizajes que allí se producen, 
es necesario reconocer un dinamismo entre los contextos transitados por los sujetos, así como los aprendizajes que allí tienen lugar. El reconocimiento de un mayor dinamismo entre contextos es una de las principales recomendaciones de este estudio para futuros diseños de investigación.

Un segundo aspecto refiere a las formas en que se analizan los conocimientos extraescolares. Pareciera que una lógica de identificación de los mismos de acuerdo a clasificaciones curriculares es la forma tradicional de definir los objetos extra-escolares a indagar, dejando en segundo lugar la indagación de otros aspectos del aprendizaje que podrían ser de relevancia para una aproximación pedagógica a las formas y sentidos que los niños y adolescentes de hoy le otorgan al aprendizaje más allá de las fronteras escolares. Consideramos que futuras investigaciones necesitan explorar otras formas de recortar los objetos a indagar. Un ejemplo que ya hemos desarrollado en esta línea es el de la conceptualización de "fondos de conocimiento", la cual ha superado la tendencia a definir los objetos en términos del curriculum escolar, y en ello reside su principal aporte.

En tercer lugar, cabe señalar la predominancia de ciertos grupos poblacionales en los objetos de estas indagaciones, donde la definición del problema de analizar las formas de aprender en distintos contextos con un interés educativo aparece recurrentemente asociada a intentos por mejorar el rendimiento escolar de alumnos provenientes de grupos desfavorecidos o minorías. Este aspecto no es menor, ya que señala en qué casos la comunidad académica identifica como útil este tipo de indagaciones. El foco puesto en poblaciones desfavorecidas o minoritarias concuerda con la hipótesis o supuesto de que para otros sectores sociales no habría tanta distancia o tantas discontinuidades entre lo que sucede en la vida escolar y lo que ocurre por fuera de ella, o quizás se trataría de un asunto no problemático. Este aspecto justifica la recomendación de realizar futuras indagaciones empíricas comparando grupos poblacionales. No obstante, resaltamos como un aspecto valioso de las investigaciones analizadas, su apuesta por proveer de conocimientos científicos que ayuden a mejorar las situaciones de aprendizaje de ciertos grupos desfavorecidos.

Por otro lado, en los estudios analizados se observan ciertos límites sistémicos en la búsqueda por recontextualizar los conocimientos cotidianos de los niños en el curriculum escolar. En primer lugar, en los intentos por promover la recuperación de los conocimientos cotidianos de comunidades desfavorecidas, se observa claramente, en cada investigación, una 
selección -muchas veces no explicitada- de ciertos conocimientos o situaciones por sobre otros, en ocasiones guiada por el interés en demostrar lo que se considera como una "riqueza" de esas experiencias familiares. Zipin (2009), por ejemplo, habla del "olvido" de los fondos de conocimiento oscuros, mientras que se favorecen aquellos "luminosos" y "positivos". Esto reclama explicitar los criterios por los cuales se mira, y por los cuales se eligen indagar ciertos aspectos y no otros.

Para finalizar, cabe advertir que los aspectos presentados en este trabajo no agotan los términos del debate ni las argumentaciones que lo sostienen. No obstante, consideramos que son mostrativos de la complejidad implícita en este problema de investigación y justifican la necesidad de sistematizar la producción académica que se ha hecho al respecto, así como el análisis crítico de las conceptualizaciones que han acompañado a estas producciones y la identificación de las vacancias o tensiones en estas conceptualizaciones. Creemos, por tanto, que este tipo de abordaje conforma un fructífero punto de partida, capaz de favorecer la construcción de nuevos puntos de llegada en lo que al desarrollo del conocimiento científico y de nuestras prácticas profesionales respecta.

\section{Referencias}

Achilli, E. (1996). Práctica docente y diversidad sociocultural. Rosario: Homosapiens.

Ada, A. F. (2003). Magical Encounter: Latino Children's Literature in the Classroom. Boston: Allyn and Bacon.

Anderson, A., Teale, W. \& Estrada, E. (2002). Experiencias de alfabetización preescolar en niños de pocos recursos: algunas observaciones naturalistas. En M. Cole; Y. Engeström; O. Vásquez (Eds.), Mente, cultura y actividad (pp. 257-270). México: Oxford University Press.

Andrews, J. \& Yee, W. (2006). Children's 'funds of knowledge' and their real life activities: two minority ethnic children learning in out-of-school contexts in the UK, Educational Review - Special Issue, 58(4), 435-449.

Bernstein, B. (1971). Class, codes and control, (Vol 1). Londres: Routledge and Kegan Paul.

Black, L. (2007). Analysing cultural models in socio-cultural discourse analysis. International Journal of Educational Research, 46, 20-30.

Borzone, A. M. y Rosemberg, C. (2000). Leer y escribir entre dos culturas. El caso de las comunidades kollas del Noroeste Argentino. Buenos Aires: Aique. 
Britten, N., Campbell, R., Pope, C., Donovan, J., Morgan, M. and Pill, R. (2002). Synthesis of qualitative research: a worked example using meta ethnography, Journal of Health Services Research and Policy, 7, 209-16.

Campbell, R.; Britten, N.; Pound, P.; Donovan, J.; Morgan, M.; Pill, R. y Pope, C. (2006). Using meta-ethnography to synthesise qualitative research. En J. Popay (ed) Moving beyond effectiveness in evidence synthesis. Methodological issues in the synthesis of diverse sources of evidence, London: National Institute for Health and Clinical Excellence

Candela, A. (2006, julio-septiembre). Del conocimiento extraescolar al conocimiento científico escolar: un estudio etnográfico en aulas de la escuela primaria. Revista Mexicana de Investigación Educativa, 11(30),797-820.

Carraher, T., Carraher, D. y Schlieman, A. (1998). En la vida diez, en la escuela cero. México: Siglo XXI.

Caruso, M. y Fairstein, G. (1997). Las puertas del cielo. Hipótesis acerca de la recepción de la psicogénesis y el constructivismo de raíz piagetiana en el campo pedagógico argentino (1950-1981). En A. Puiggrós (dir.) Dictaduras y utopías en la historia reciente de la educación argentina (1955-1983), (pp. 157-220). Buenos Aires: Galerna,

Cazden, C. (1991). El discurso en el aula. El lenguaje de la enseñanza y del aprendizaje. Paidós: Barcelona.

Davis, P. (2007). How cultural models about reading mediate classroom (pedagogic) practice. International Journal of Educational Research, 46, 31-42.

Edwards, D. \& Potter, J. (1992). Discursive psychology. Londres: Sage.

Edwards, V. (1995). Las formas del conocimiento en el aula. En E. Rockwell (coord.) La escuela cotidiana (pp. 145-172). México D. F.: Fondo de Cultura Económica

Elichiry, N., Scavino, C. y Arrúe, C. (2005). Deberes escolares ¿un nexo entre la familia y la escuela?. Revista Argentina de Educación, 26 (29), 87-100.

Elichiry, N. (2007). Aprendizajes y contextos de práctica (Parte III) .En D. Aisenson, J. Castorina, N. Elichiry y A. Lenzi (Comps.). Aprendizaje, Sujetos y Escenarios. Investigaciones y prácticas en psicología educacional. Buenos Aires: Noveduc.

Elichiry, N, Maddonni, P., Scavino, C., Aizencang, N., Arrúe, C., Bendersky, B.,... Sanchez, A. (2008). Sujetos, familias y escuelas: continuidades y discontinuidades en los aprendizajes cotidianos (pp. 228-229) Ponencia presentada en XV Jornadas de Investigación y Cuarto Encuentro de Investigadores en psicología del MERCOSUR. Ciudad Autónoma de Buenos Aires. 
Epstein, J. \& Dauber, S. (1991). School programs and teacher practices of parent involvement in inner-city elementary and middle schools. The Elementary School Journal,91,1523.

Ferreiro, E. (1986). El cálculo escolar y el cálculo con dinero en situación inflacionaria. En Proceso de alfabetización. La alfabetización en proceso (pp. 105-133).Buenos Aires: Centro editor de América Latina.

Ferreiro, E. y Teberosky, A. (1979). Los sistemas de escritura en el desarrollo del niño. México D. F.: Siglo XXI.

Gandulfo, C. (2007). Entiendo pero no hablo. El guarani "acorrentinado” en una escuela rural: usos y significaciones. Buenos Aires: Antropofagia.

González, N., y Moll, L. (2002). Cruzando El Puente: Building Bridges to Funds of Knowledge. Educational Policy, 16 (4), 623-641.

Greenberg, J. (1989). Funds of knowledge: historical constitution, social distribution, and transmisión. Paper presented at the annual meetings of the Society for Applied Anthropology, Santa Fe, NM.

Gough, D. (2004). Systematic Research Synthesis to inform the development of policy and practice in education. En G. Thomas y Pring, R. (eds): Evidence-based practice. Buckingham: Open University Press (pp 44-62).

Gough, D y Elbourne, D. (2002). Systematic research synthesis to inform policy, practice and democratic debate, Social Policy and Society, 1, 1-12

Gumperz, J. (Ed.). (1984).Introduction. Language and Social Identity. Cambridge, ma: Cambridge University Press.

Heath, Sh. B. (1983). Ways with words. Language, life and work in communities and classrooms. Cambridge: Cambridge University Press.

Huddleston, A. (2003, invierno). Incorporating Popular Culture into the Curriculum.Educational Horizons, 16, 95- 97.

Hughes, M. (2006a). End of Award Report: Home-School Knowledge Exchange and Transformation in Primary Education. Londres: ESRC-TLRP. Recuperado de http://www.esrcsocietytoday.ac.uk

Hughes, M. (2006b). Home-school knowledge exchange. Educational Review, 58 (4), 385487.

Isbell, D. (2006). Popular music and the public school music classroom. Update: Applications for Research in Music Education, 26 (1) 
Jasis, P. \& Ordoñez-Jasis, R. (2004-2005, Diciembre/Enero). Convivencia to empowerment: Latino Parent Organizing al La Familia. The High School Journal, 88(2), 32-42.

Kalman, J. (2003). El acceso a la cultura escrita: la participación social y la apropiación de conocimientos en eventos cotidianos de lectura y escritura. Revista Mexicana de Investigación Educativa, 17 (8), 37-66.

Kalman, J. (2008). Discusiones conceptuales en el campo de la cultura escrita. Revista Iberoamericana de la educación, 46. Obtenido el 8 de Febrero de 2010 de: http://www.rieoei.org/rie46.htm

Labov, W. (1969). The logic of non-standard English. En J. E. Alacis (Ed.) Report of the Twentieth Round Table Meeting on Linguistics and Languaje Studies. Colección Monografías sobre Lengua y Lingüítica.Washington: Georgetown University Press, 22, 129.

Lacasa, P., Gómez, M., Reina, A., Cosano, C. (1996-1997). Saltando entre el hogar y la escuela. Aprendiendo a leer y escribir. Kikiriki, 42-43,37-43

Levy, E. (2010). Reportaje a Peter Sloterdijk. Una filosofía contra el rencor. Revista $\tilde{N}$, Clarín. Obtenido el 3 de Junio de 2010 de:

http://www.clarin.com/suplementos/cultura/2003/06/28/u-00211.htm

Limón, M., y Carretero, M. (1997). Las ideas previas de los alumnos ¿Qué aporta este enfoque a la enseñanza de las ciencias? En M. Carretero (1997). Construir y enseñar. Las ciencias experimentales. Buenos Aires: Aique.

Marsh, J. (1999). Batman and Batwoman go to school: popular culture in the literacy curriculum. International Journal of Early Years Education,7 (2), 117- 131.

Martin-Jones, M., \& Saxena, M. (2003). Bilingual resources and 'funds of knowledge' for teaching and learning in multi-ethnic classes in Britain. International Journal of Bilingual Education and Bilingualism, 6 (3-4).

Moll, L., \& Greenberg, J. (1990). Creating zones of possibility. En L. Moll (Ed.). Vygotsky and Education: Instructional implications and applications of sociohistorical psychology, (pp. 319-348), Cambridge University Press.

Moll, L., Amanti, C., Neff, D., González, N. (2005). Funds of knowledge for teaching: Using a qualitative approach to connect homes and classrooms. En N. González, L. Moll \& C.Amanti (Eds.). Funds of knowledge: Theorizing practices in households, communities, and classrooms (pp. 71-88). Mahwah, NJ: Lawrence Erlbaum Associates, Inc.

Moriana, J. A., Alós, F., Alcalá, R., Pino, M. J., Herruzo, J. y Ruiz, R. (2006). Actividades extraescolares y rendimiento académico en alumnos de Educación Secundaria. Elec- 
tronic Journal of Research in Educational Psychology, 8(4), 35-46. Obtenido el 4 de diciembre de $\quad 2010 \quad$ en http://www.investigacionpsicopedagogica.org/revista/articulos/8/espannol/Art_8_82.pdf

Nakache, D. (2007). La tele -entre la familia y la escuela- En D. Aisenson, A. Castorina, N. Elichiry, A. Lenzi y S. Schlemenson (Comps) Aprendizajes, sujetos y escenarios. Buenos Aires: Noveduc.

Noblit, G. and Hare, R. (1988) Meta-ethnography: synthesizing qualitative studies. Newbury Park: Sage.

Paradise, R. (1990). Pasos hacia la comunicación intercultural: las adaptaciones de niños mazahuas y sus maestros en la práctica dentro del aula. Trabajo expuesto en el Seminario de Cultura y Educación, Seminario para la cultura, Consejo Nacional para la Cultura y las Artes, México.

Paradise, R. (1991). El conocimiento cultural en el aula: niños indígenas y su orientación hacia la observación. Infancia y Aprendizaje, 55, 73-85.

Paradise, R. (1994). The autonomous behavior of indigenous students in classroom activities.En A. Álvarez y P. del Río (Coords.). Education as cultural construction (pp. 8995). Fundación Infancia y Aprendizaje: Madrid.

Peralta, F. J. y Sánchez, M. D. (2003). Relaciones entre el autoconcepto y el rendimiento académico, en alumnos de Educación Primaria. Electronic Journal of Research in Educational Psychology, 1(1), 95-120. Obtenido el 5 de octubre de 2009 en http://www.investigacionpsicopedagogica.org/revista/articulos/1/espannol/Art_1_7.pdf

Philips, S. (1972). Participant structures and communicative competence: Warm Springs children in community and classroom. En C. Cazden(Comp.) Functions of language in the classroom (pp. 370-394).Nueva York: Teacher's College Press.

Philips, S. (1982). The invisible cultura. Communication in classroom and community on the Warm Springs Indian Reservation. Nueva York: Longman.

Popay, J. (2006) Moving beyond effectiveness in evidence synthesis. Methodological issues in the synthesis of diverse sources of evidence, London: National Institute for Health and Clinical Excellence.

Prado, M., Canigia, M., y Gunset V. (2009, julio 1-3). Los otros y nosotros en la autorización de la palabra: desarrollo de competencias comunicativas y prácticas docentes en contextos de pobreza. Ponencia presentada en VI Jornadas de Investigación en educación: 
investigación, conocimiento y protagonismo de los actores en el campo educativo. Facultad de Filosofía y humanidades. Universidad Nacional de Córdoba. Córdoba.

Rockwell, E. (1992). Tales from Xaltipan: Documenting Orality and Literacy in Rural Mexico En Cultural Dynamics. Leiden, Países Bajos: E. J. Brill, 5(2),156-175.

Sagastizabal, M. A. (2000). La escuela toba de Rosario. Reuniendo datos para el diagnóstico.En Diversidad cultural y fracaso escolar. Educación intercultural: de la teoría a la práctica. Rosario:Ediciones IRICE.

Thomson, P. (2002). Schooling the rustbelt kids. Making the diff erence in changing times. Sydney: Allen \& Unwin (UK: Trentham Books).

Thomson, P., \& Hall, Ch. (2008). 'Opportunities missed and/or thwarted? 'Funds of knowledge' meet the English national curriculum'. Curriculum Journal, 19 (2), 87-103.

Wells, G. (1986). La experiencia del lenguaje de niños de cinco años en la casa y en la escuela. En La construcción social de la alfabetización (pp.85-108).Temas de educación Paidós MEC.

Zipin, L. (2009). Dark funds of knowledge, deep funds of pedagogy: exploring boundaries between lifeworlds and schools. Discourse: Studies in the Cultural Politics of Education, 30 (3) 317-331. 